\title{
Improving the Effectiveness of Compute-based Educational Game with IMS Learning Design
}

\author{
Miao-miao Zeng \\ Education Institute, ZhaoQing University, ZhaoQing, China \\ àmiao.m@163.com
}

Keywords: Computer-based Educational Game (CEG), IMS Learning Design (LD), Activity theory, Elements, Structure.

\begin{abstract}
This article employed the concept of IMS learning design (LD) to realize the balance of "educational function" and "entertainment" in Computer-based educational game (CEG). A design structure of CEG based on activity theory was set up firstly, and then a method how to use LD to model each element in CEG was described, which was illustrated by an integration case as well. Finally, the conclusion that LD used as a framework of game activity design can improve the effectiveness of CEG was got.
\end{abstract}

\section{Introduction}

Computer-based educational game (CEG) is a new and exciting area which in virtue of the environment created by computer. It has been introduced into education as an alternative to the traditional way of teaching. It is both fun and informative [1]. CEG can provide environments that we immerse ourselves in. It can motivate students learning actively, making learning fun, effective and efficient. Currently, teachers sometimes use games in their lesson plans (Squire, 2005). However, the use of games is often leading to disconnect between educational setting and game [2]. There are not many CEGs of good educational quality for learning.

How can game designers create opportunities for deep learning without sacrificing immersive game play and enjoyment? Now, there is no tool can regulate and realize the balance of "educational function" and "entertainment" in CEG, the concept of IMS learning design may be an available method and notion, which can provide a framework of design to improve the effectiveness of CEG.

\section{Understanding the Design Structure of CEG}

Introduction of IMS Learning Design. IMS Global Learning Consortium Inc. (IMS) released the Version 1.0 Learning Design specification in early 2003. This specification (IMS Learning Design) aims to represent the learning design of "units of learning" in a semantic, formal and machine interpretable way [3] (Koper \& Olivier, 2004). In a word, LD can not only describe learning content, but also describe learning activities between learners and teachers, it mainly focused on learning activities and processes [4].

LD specification is divided into three parts. Level A provides the basic functionality to define roles, resources and activities; Level B adds properties and conditions, which support sophisticated adaptability and interactivity; Level $\mathrm{C}$ provides support for notification so that teachers, learners and other e-Learning systems can be informed of progress in the learning activities [5].

The Design Structure of CEG. According to the activity theory and system theory, the design of the game can be regarded as an activity system as well. Broadly defined, activity theory is a philosophical and cross-disciplinary framework for studying different forms of human practices as development processes. [6]. This model divides activity into six elements and three levels based on systematic analyze: subject, rule, community, object, instrument and division of labor. It provides an available theory framework for CEG, while it provides a suitable architecture for the design of game. The essence of CEG is systematic activity. Correspondingly, the structure of CEG can be described as Fig.1. It represents the CEG activity system also contains six elements: player, group, tool, task, rule, and division of task. The outcome is to promote learning. 


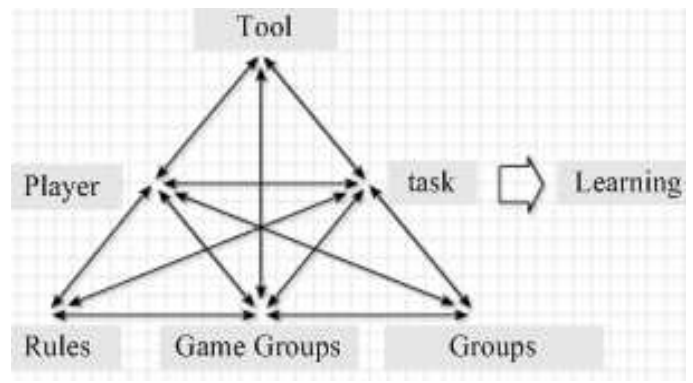

Fig 1. Structure of CEG.

The description of these elements can be listed in table 1. It expresses the basic feature and characteristic of these elements in compute-based educational game.

Table 1. Description of Elements in an Educational Game Activity

\begin{tabular}{l|l}
\hline Elements in CEG & \multicolumn{1}{|c}{ Description } \\
\hline Player & $\begin{array}{l}\text { The executants of the game, the subject engaging in the game. } \\
\text { The collection of tasks that the player should and willing to } \\
\text { complete } \\
\text { The referenced entities when playing the game. }\end{array}$ \\
Tool & $\begin{array}{l}\text { A definition of the responsibilities each player has to shoulder in } \\
\text { achieving the appointed goal. } \\
\text { The regulation that the player should obey. } \\
\text { Division of task }\end{array}$ \\
Rule & $\begin{array}{l}\text { A community composed of various players. } \\
\text { players during the process. }\end{array}$ \\
Group &
\end{tabular}

As expatiated above, a CEG can be regarded as an activity system. While IMS LD focused on learning activity and process, the generality of IMS LD implies that it should also be capable of modeling CEGs [7]. Therefore, we can try to employ the concept of LD to design CEG. In fact, some researchers have proved that LD can provide a conceptual and practical framework supporting the development of CEG [8].

\section{Using LD to Model CEG}

Taking into account the previous description, a correspondence can be established between them and the level to be modeled with LD (Table 2).According to system theory, the analyzing of elements in CEG is fundamental. This section will analyze how to use LD to design the components of CEG in detail, further hold the essence and development of the whole game to improve the effectiveness.

Table 2. Correspondence between Elements of LD and the Game Activity System

\begin{tabular}{c|c|c}
\hline Elements in game & Element in IMS LD & Level \\
\hline Player & Role & $\mathrm{A}$ \\
Task & Activity & $\mathrm{A}$ \\
Tool & Environment & $\mathrm{A}$ \\
Division of task & Role-parts & $\mathrm{A}$ \\
Rule & Activity-structure/condition & $\mathrm{B}$ \\
Group & Role/Properties & $\mathrm{B}$ \\
Interaction/Feedback & Notification & $\mathrm{C}$ \\
\hline
\end{tabular}

Elements of CEG Modeled in Level A. As in table 2, there are four elements in CEG that can be modeled in level A. While rule and group can be encoded by two elements (level A/ B), we will put it to level B. 
Player and Role. The player in a game is the subject i.e. who is playing? At present, the players are mostly students in CEG. However, the teacher hardly participates in the process, so it is lack of management and monitor. Role in LD allow the types of participants in CEG be specified. There are two basic role types: Learner and Staff [7]. These can be sub-typed to allow learners to play different roles (As showed in Fig.2).

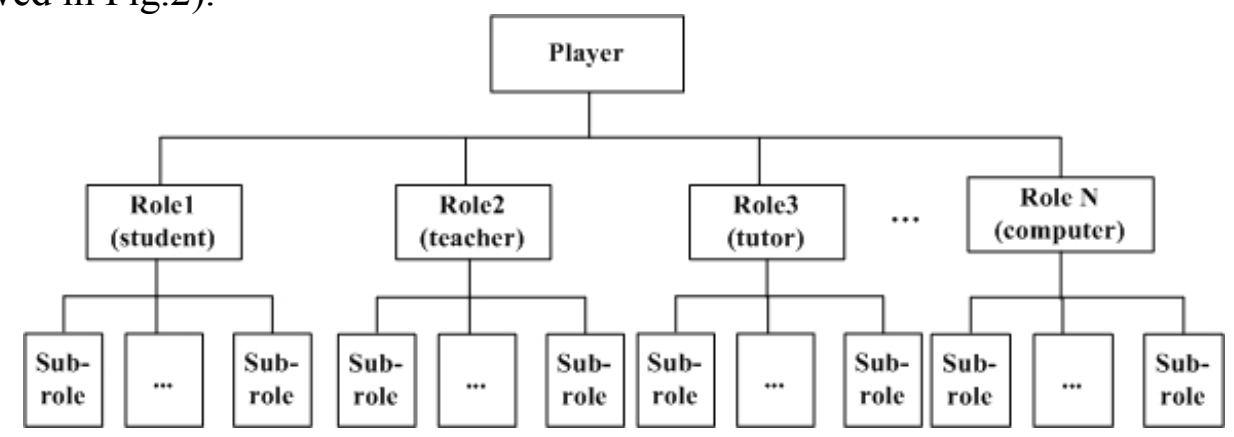

Fig 2. Structure of Players Modeled in LD

Therefore, we can use LD to specify the roles and numbers in CEG flexibly. In a game, it can have many different roles; as to the same role, it can set sub-role to define different level, but the division varies and each role is granted different right. It is a great progress that introducing teacher into CEG as a mentor or guider, which can monitor the process better to improve the impact of game.

Task and Activity. Task is the core and key of CEG. It is what the player must complete; it may be a single assignment or collection of a series of assignments and missions. There is a corresponding element "activity" in LD. Activity is one of the core structural elements for LD. They form the link between the roles and the learning objects and services in the learning environment.

It is possible to employ activity to realize the design of task, we can establish the learning activity as the primary mission, what is must complete. When is playing, the support activity may support or help players. Introducing activity attribute to CEG will make it more friendly and personalized, the players can get support when need, which make the player be happy and willing to complete the task.

Tool and Environment. In order to accomplish the goal of the game, the players must use suitable tools in playing process. Tool is the referenced entities to achieve goal when playing games. The tool connects the players and tasks; it is the medium in game activity. Generally speaking, the kind of game varies, the tools available are different.

In LD, Activities take place in a so-called "environment", which is a structured collection of learning objects, services, and sub-environments [7]. Current service types in LD are send-mail, conference, monitor, and index search. Environment allows monitoring any kind of property assigned to a user or a role, the selection of services to be included needs to be driven by the community. The environment attribute in LD can be used to model tool in the structure of CEG, though it may be not enough to satisfy the need of the designer, LD specification can integrate other specifications.

Division of task and Role-parts. Division of task in a game is the process of transforming the goal of one game to the result of another activity, with a defining of the responsibilities each player has to shoulder in achieving the appointed goal. In LD, role-part can be used to model it. The core part of the learning design is represented in the "play". A play is modeled according to a theatrical play. In general: a play consists of a sequence of acts. In each act, different activities are set for different roles, role-parts is the mechanism that allows more than one role to perform at the same time.

Role-parts enable several users, playing the same or different roles, to participate in the same act [7]. Each role-part associates exactly one role with one activity or with one environment. Multiple role-parts within one act are performed concurrently. With role-parts, the responsibilities and tasks of each player are defined more clearly.

Elements of CEG Modeled in Level B.

Rule and Activity-structure/Condition. Rules are regulations pre-designed according to the goals of games. The player's behaviors are severely restricted by the game rules. That is to say, every game has its special rules, which have absolute sanctions on all the players. The rule reflected to LD is 
activity-structure, which provides the mechanisms to structure activities. In addition, activity-structure contains activity reference; it referenced the environment in which the activity must be executed. This allows for learning design models where a series of different activities are performed within the same environment.

Condition is another attribute in LD helping to design the rules. Condition is mostly useful within activity-structure of the type "selection". The activity-structure and condition in LD is similar to the rule in CEG. By defining activity-structure and condition, we can reference environments and conditions of completion, connect the roles (players in CEG) and environments (tools in CEG). In brief, activity-structure and condition can specify the activity flow of a game, can constrain and guide the players when, use which tool, in what sequence, when the game finishes and so on.

Group and Properties. A group is a community composed of various roles taken by different player. Currently, much attention is required to the importance of team analysis and the complexity of each role property within each specific team. Shaban and Head (2003) have called the use of group projects an essential tool for learning. A group in CEG is both task-related and social-emotional in nature.

LD Level B introduces properties, which form the basis on which to build user and role dossiers and portfolios. Properties are an essential part of monitoring, personalization, assessment and for user-interaction [7]. A communication between players can be established between both using specific features of this language and the system of properties, and global elements. In addition, at runtime more than one user can be assigned to the same role. In this sense, roles may be used for grouping purposes. The utilization of role and properties can set variables to each player.

Elements of CEG Modeled in Level C.As showed in Table 2, the elements Interaction/Feedback can be modeled by two attributes: notification and properties separately in level B and C.

Interaction /Feedback and Notification/Property. Interaction/Feedback is the interrelations and different reactions showed by different players during the process of their using some given tools to achieving the goal. The arrowhead in Fig. 1 is just a marker of this interaction/feedback mechanism. However, here with only one player involved, it can only indicate the relations between this player and other participating factors.

Level C introduces notification, which can be used to promote interaction. It is possible to send a message to a role or to assign new activities based on certain events [9]. The message may be a dialogue box with a selection of questions, such as "Great, you are right!" "Wrong! Do you want to try again?" "Do you know why?" and so on. These reflections act as a pedagogical agent, which adds a new dimension by supporting real-time event-driven learning flow. More generally, it enables the automation of learning flow in CEG, which are triggered by the completion of tasks. The notification realizes adaptability and intelligence to some extent.

\section{An Integration Case — “Memory"}

In order to illustrate the previous analysis; a game called Memory has been chosen to support the integration of IMS LD and CEG. Memory is a card game. It was scripted and programmed in Flash/Action Script by Burgos, further on it has been adapted to XML and LD in the OUNL [10]. This Fig 3 is about the user interface of Memory [10]. 


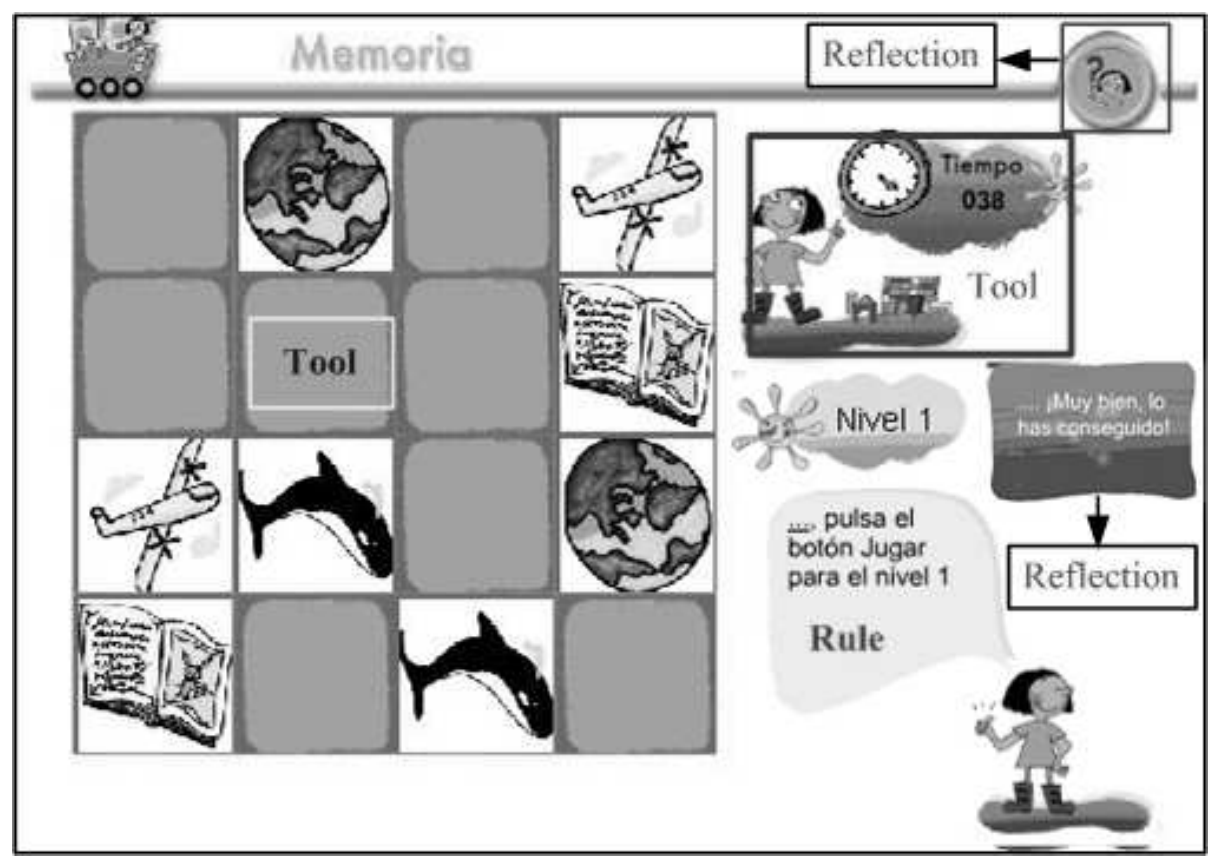

Fig 3. Compute-based Educational Game Memory in IMS LD

We can analyze this case according to the structure of game activity (Fig1):

Player: This game contains two or more players.

Tool: The tool in Memory includes materials (cards) and a flat surface; there is also a timer and navigation.

Task: the task is divided into three steps: Preparation, Play, and Evaluation.

Rule: the player should press the button "Jugar" to start, then turns over first one card and then another. If the face values pair up, then the cards are removed to the player's score pile and the player takes another turn.

Reflection: When finished, the agent will congratulate you, which is developed by notification and condition.

Group and Division of task: They are not defined definitely in this game.

Though simple, the game expressed the notion of applying LD into CEG well; this game is both educational and fun. As to educational features, the student can control the flow of game freely, and obtain support/feedback from different roles, the process of playing/leaning can be recorded to monitor; As to entertainment the game offers friendly and colorful interface, the learning content is in the form of meaningful card.

\section{Discussions and Conclusions}

It is a new attempt and method to use LD to model CEG; maybe it will bring a brand-new area as well. It is not only feasible, great benefits can also be attained by employing LD into CEG, as follows (though there are some shortcomings):

The separation of activity structure from its content makes the reuse of existing game module possible. Moreover; the LD-encoded game is transportable [8]. When a game activity is encoded as in LD; it can be inspected to see its organization and pedagogy within the context of the module. The element of level B and level C in LD can be used to add the adaptability and intelligence of learning in CEG. Beginning with elements of CEG, the integration can prove the effectiveness of educational game based on system theory.

In conclusion, each component in CEG can be modeled in LD in virtue of some other relative specifications. Especially the level $\mathrm{B} / \mathrm{C}$ is basic to manage all the elements in $\mathrm{CEG}$. This paves way for adaptive learning and role-play and simulations. It can improve the effectiveness of CEG, leading to active and effective learning. 


\section{References}

[1] Tollefsrud: The Educational Game Editor: the Design of a Program for Making Educational Computer Games. Trondheim(2006). Retrieved November14,2006

http://www.diva-portal.org/diva/getDocument?urn_nbn_no_ntnu_diva-737-1_fulltext.pdf

[2] Daniel Burgos, Tattersall and Koper: Re-purposing existing generic games and simulations for e-learning (2005) Retrieved September14, 2006, http://dspace.learningnetworks.org

[3] Koper \& Olivier: Representing the Learning Design of Units of Learning, Educational Technology \& Society (2004)

[4] Rob Koper: Current Research in Learning Design. Educational \& Society, Vol 9 NO 1 (2006), p.13-22

[5] Sandy: A Review of Learning Design. JISC Project, Britain (2004)

[6] Kari Kuutti: Activity Theory as a potential framework for human computer interaction research .Activity Theory and Human Computer Interaction, Cambridge (1995) , p. 17-44

[7] IMS Learning Design Specification (2003): IMS Global consortium. Retrieved October 8th, 2006, http://www.imsglobal.org.

[8] Burgos, Tattersall, Koper: Can IMS Learning Design be used to model computer-based educational games? (2006) Retrieved September14 http://dspace.learningnetworks.org

[9] Koper, R., Tattersall, C.(Eds) :Learning Design: A Handbook on Modelling and Delivering Networked Education and Training. Germany: Springer Verlag (2005), p.231

[10]Burgos, D., Berbegal, N., Griffiths, D. (2005a): “IMS Learning Design Level 0". Retrieved November 7th, 2006 http://moodle.learningnetworks.org/mod/resource/view.php?id=17 